\title{
Preliminary report on the effects of vagus stimulation on the dog's stomach and the influence of asphyzia on these effects.
}

BY Z. BERCOVITZ.

[From the Hull Physiological Laboratory of the University of Chicago and Physiological Laboratory of Baylor Medical College,

$$
\text { Dallas, Texas.] }
$$

In a previous report ${ }^{1}$ attention was called to the fact that in the turtle repeated vagus stimulation was followed by a progressive decrease in the gastric response to each stimulation. It was further pointed out in another report ${ }^{2}$ that the stomach could not be tetanized by prolonged vagus stimulation, also that reduction in the temperature of the turtle was followed by a decreased gastric response to vagus stimulation. These facts seemed to indicate that in the turtle a complex neuro-muscular mechanism controlled the response of the stomach to vagus stimulation.

The object of this study therefore was to determine if the dog's stomach would respond to vagus stimulation in the same manner as the turtle's stomach.

\section{METHODS.}

The dogs used in this study were decerebrated in order to avoid the depressing influence of an anesthetic.

The balloon method was used for recording gastric contractions. The balloon was introduced into the stomach through $(a)$ healed gastric fistula; $(b)$ through slit in the anterior wall of the stomach near the pylorus; $(c)$ through the mouth and esophagus; $(d)$ through the duodenum. The results were the same in all cases.

In most cases a simultaneous blood pressure tracing was made from the carotid artery and in others the chest was opened, artificial respiration given and the heart observed directly.

To produce asphyxia of the stomach a lifting ligature was placed under the thoracic aorta.

The animals were cooled by packing them in cracked ice until the rectal temperature dropped to the desired point. As a rule $I \frac{1}{2}$ to $2 \frac{1}{2}$ hours were required to cool the decerebrated $\operatorname{dog}$ to $23^{\circ} \mathrm{C}$.

' Bercovitz and Rogers, Amer. Journ. Physiol., 1921, 1v, 323.

2 Rogers and Bercovitz, Amer. Journ. Physiol., I921, lvi, 257. 


\section{Results.}

Effect of Repeated Vagus Stimulation.-In the dog stimulations of the vagus with a tetanizing current repeated at short intervals with a given strength of current are followed by contractions of the stomach of approximately uniform amplitude. Complete cardiac inhibition was observed during each stimulation. The apparent ability to produce an artificial rhythm of the dog's stomach by repeated stimulations of the vagus is in striking contrast to the rapid failure of gastric contractions on repeated vagus stimulation in the turtle.

Effect of Prolonged Vagus Stimulation.-Prolonged stimulation of the vagus with a tetanizing current of moderate strength and also a strong tetanizing current is followed by only a single contraction of the stomach and its usual subsequent relaxation in spite of continued stimulation. Weak peristalses in the pyloric region may occur after the one contraction during the balance of the stimulation but usually stops shortly after the stimulation has ceased. There is no indication of a tetanus or increased tone of the stomach. These results are similar to those obtained in the turtle.

It was noted from the blood-pressure tracings and also from direct observation of the heart that complete vagus inhibition of the heart failed at about the same time as the relaxation of the stomach began. It would seem that the mechanism operating to prevent gastric tetany is the same as that which prevents prolonged complete cardiac inhibition from vagus stimulation.

Influence of Cooling.-In dogs cooled to $25^{\circ} \mathrm{C}$. to $23^{\circ} \mathrm{C}$. it was found that repeated stimulations of the vagus were followed by no indications of either failure of gastric response or increased or permanent tone as the result of the stimulations. Prolonged vagus stimulation at this temperature is not followed by tetanus of the stomach.

It was further noted that at a temperature of $21^{\circ} \mathrm{C}$. there was a failure to obtain a gastric response from vagus stimulation but stimulation of the stomach wall was followed by a contraction. No cardiac inhibition was noted at this temperature in response to vagus stimulation. As the temperature was raised there was a corresponding increase in gastric response to stimulation of the vagus. 
The heart seemed to be more easily thrown into fibrillation in the animals which had been cooled than in correspondingly prepared animals at normal body temperature. Death in most of the experiments on cooling was due to fibrillation of the heart.

Influence of Temporary Asphyxia.-In dogs with the thoracic aorta ligated repeated stimulations of the vagus are followed by rapid failure of gastric response to stimulation. Allowing the blood to pass again to the stomach is followed by recovery of the gastric response to vagus stimulation.

These experiments would seem to indicate that in the dog as well as in the turtle a complex neuro-muscular mechanism influences the effects of vagus stimulation on the stomach. This mechanism in the dog is very sensitive to partial asphyxia. It may be in the turtle that the rapid failure of gastric response to vagus stimulation is dependent on circulatory changes causing an asphyxia of the complex neuro-muscular mechanism.

\section{SUMmaRY.}

I. In the dog stimulations of the vagus repeated at short intervals are followed by contractions of the stomach of approximately uniform amplitude.

2. Prolonged stimulation of the vagus is followed by a single contraction of the stomach and its usual subsequent relaxation in spite of continued stimulation. Failure of cardiac inhibition occurs at about the same time as relaxation of the stomach begins.

3. Reduction of the body temperature to $25^{\circ} \mathrm{C}$. to $23^{\circ} \mathrm{C}$. is followed by decreased response to vagus stimulation but no indications of either failure of gastric response to repeated vagus stimulation or tetanus. At $2 \mathrm{I}^{\circ} \mathrm{C}$. vagus stimulation is without effect on the stomach but stimulation of the stomach wall is followed by a contraction.

4. Partial asphyxia of the stomach by shutting off the blood supply to the part is followed by rapid failure of gastric response to repeated vagus stimulations similar to that observed in the turtle. Removal of the asphyxia is followed by recovery of the gastric response to stimulation. 